\title{
FETAL IMPACT WITH THE USE OF PHENYLEPHRINE INTRAVENOUS INFUSION USED AS PROPHYLAXIS OF ARTERIAL HYPOTENSION AFTER INTRADURAL ANESTHESIA AT ELECTIVE CESAREAN
}

GIL BONA J., PEÑA CALVO P., ROVIRA FLORES E., BORQUE MARTIN JL., MARTÍNEZ UBIETO J., MUÑOZ RODRÍGUEZ L. DEPARTMENT OF ANESTHESIOLOGY. MIGUEL SERVET HOSPITAL. ZARAGOZA.

\section{OBJECTIVE}

To assess the fetal repercussion, both in the Apgar test and in cord blood gas analysis, of the use of continuous intravenous infusion of phenylephrine used as prophylaxis for hypotension after intradural anesthesia in elective cesarean

\section{MATERIAL AND METHOD}

We performed a prospective study including all elective cesarean performed at the obstetrics service of the Miguel Servet University Hospital from November 2012 to March 2015. We excluded the non-acceptance of participation in the study, the presence of uterine dynamics or the suspicion of maternal-fetal pathology. 105 patients were included into the study.

All patients underwent an intradural anesthesia with $0.5 \%$ hyperbaric bupivacaine and doses according to the size (<155cms-10mg, 155-165-11mg,> 165-12mg)

All newborns were submitted to an apgar test at 5 minutes of life, as well as arterial and venous blood from umbilical cord was taken for determination of gasometry.

We made a comparative analysis evaluating pre and post intradural anesthesia data according if patients were administered a continuous intravenous infusion of phenylephrine $(2 \mathrm{mcg} / \mathrm{Kg} / \mathrm{min})$ (53 patients)or not( 52 patients). The patients were randomly assigned to each group.

To correlate different variables we used the chi square of Pearson or Fisher test for discrete variables and t-student or $U$ Mann-Whitney test for continuous variables. Significance at $p<0.05$

\section{RESULTS}

\section{DATA}

PRE INTRADURAL ANESTHESIA

Age (years)

$\mathrm{BMI}(\mathrm{Kg} / \mathrm{m} 2)$

Gestational age(weeks)

POST INTRADURAL ANESTHESIA

Fetal extraction time(sec)

Apgar after 5 minutes $=9$

Apgar after 5 minutes $=10$

Umbilical cord arterial ph

Umbilical cord arterial pCO2

Umb.Cord art. Lactic acid

Umb.Cord art. Glucose

Umb.Cord art. $\mathrm{HCO} 3$

Umbilical cord arterial SO2

Umbilical cord venous ph

Umbilical cord venous pCO2

Umb.cord ven. Lactic acid

Umb.cord ven. Glucose

Umb.cord ven. $\mathrm{HCO} 3$

Umbilical cord venous SO2

\begin{tabular}{l} 
Control group ( \\
$34.1(4.8)$ \\
$27.4(4.1)$ \\
$38(37-39)$ \\
\\
$73.5(45-105)$ \\
$3(6 \%)$ \\
$48(94 \%)$ \\
$7.29(7.25-7.33)$ \\
$54.5(52-59)$ \\
$2.05(1.7-2.9)$ \\
$50(46-56)$ \\
$26(25.2-27.3)$ \\
$6(3-13.5)$ \\
$7.37(7.31-7.39)$ \\
$43(39-47)$ \\
$1.7(1.5-2.1)$ \\
$61(57-69)$ \\
$24.1(23.5-24.8)$ \\
$29(14-47)$ \\
\hline
\end{tabular}

\begin{tabular}{|l|l|}
\hline Prophylaxis group(n=53) & $\mathbf{P}=$ \\
$33.7(4.8)$ & $0.657^{\mathrm{a}}$ \\
$28.2(4.1)$ & $0.344^{\mathrm{a}}$ \\
$38(37-39)$ & $0.385^{\mathrm{b}}$ \\
& \\
$68(46.5-87.5)$ & $0.582^{\mathrm{b}}$ \\
$4(7 \%)$ & $0.735^{\mathrm{b}}$ \\
$49(93 \%)$ & $0.735^{\mathrm{b}}$ \\
$7.29(7.26-7.32)$ & $0.690^{\mathrm{b}}$ \\
$57(55-61)$ & $0.018^{\mathrm{b}}$ \\
$1.7(1.5-2.4)$ & $0.022^{\mathrm{b}}$ \\
$50(46-55)$ & $0.705^{\mathrm{b}}$ \\
$27.6(26.2-28.4)$ & $0.003^{\mathrm{b}}$ \\
$8(3.5-12)$ & $0.746^{\mathrm{b}}$ \\
$7.35(7.31-7.39)$ & $0.377^{\mathrm{b}}$ \\
$45(41-49)$ & $0.252^{\mathrm{b}}$ \\
$1.6(1.3-1.9)$ & $0.053^{\mathrm{b}}$ \\
$60(56-64)$ & $0.145^{\mathrm{b}}$ \\
$24.3(23.6-25.4)$ & $0.313^{\mathrm{b}}$ \\
$27(18-43)$ & $0.933^{\mathrm{b}}$ \\
\hline
\end{tabular}

a- Values expressed as means (+/- typical deviation) and compared using the Student's T-test

b- Values expressed as medians (interquartile range) and compared using the U-Mann-Whitney test

\section{CONCLUSIONS}

Prophylaxis of arterial hypotension with phenylephrine in intravenous infusion at $2 \mathrm{mcg} / \mathrm{kg} / \mathrm{min}$, did not present a significant impact at the fetal level, assessed by Apgar test and cordometric gasometry. 